\title{
Implementation of a New Method to Quantify the Clinical Activity in a Comprehensive Cancer Center
}

\author{
César Muñoz ${ }^{1,2, \dagger, ~ *, ~ R a f a e l ~ A ́ l v a r e z ~}{ }^{1,2, \dagger}$, Jesús Rodriguez ${ }^{1,2}$, Lisardo Ugidos $^{1,2}$, Enrique Sanz ${ }^{1,2}$, \\ Antonio Cubillo ${ }^{1,2}$ \\ ${ }^{1}$ Centro Integral Oncológico Clara Campal (CIOCC), Hospital Universitario HM Sanchinarro, Madrid, Spain \\ ${ }^{2}$ Department of Medical Sciences, CEU San Pablo University, Madrid, Spain
}

Email address:

cgregorio@hmhospitales.com (C. Muñoz)

${ }^{*}$ Corresponding author

$\uparrow$ César Muñoz and Rafael Álvarez are co-first authors.

\section{To cite this article:}

César Muñoz, Rafael Álvarez, Jesús Rodriguez, Lisardo Ugidos, Enrique Sanz, Antonio Cubillo. Implementation of a New Method to Quantify the Clinical Activity in a Comprehensive Cancer Center. Journal of Cancer Treatment and Research. Vol. 7, No. 1, 2019 , pp. 9-12. doi: $10.11648 /$ j.jctr.20190701.12

Received: January 9, 2019; Accepted: March 1, 2019; Published: March 21, 2019

\begin{abstract}
There is no generalized way of evaluating clinical care activity in a comprehensive Cancer Center. Time clinical units is the most common procedure worldwide. In a spanish group it has been developed a new system based on the daily real activity of each medical oncologist of his team. In adittion, it has been collected the daily activity from January 2016 to December 2017 of each doctor considering different values (from 1 to 4) depending on the complexity of the activity. Follow up visit of a patient [1], treatment visit [2], Clinical trial visit or Inpatient visit (3) and New Patient First visit (4). Then it was added all the daily values of each medical oncologist. Moreover, the clinical activity of each Oncology Unit changed clearly when it was applied the new method. For example, breast cancer unit change from $24 \%$ to $21 \%$ of the global activity, GI Unit from $47 \%$ to $49 \%$, Lung Unit from 13\% to 14\%. In Genitourinary and Gynecological cancer and Prostate tumor units there are no change. These changes draw the different complexity of each Oncology Unit. It was clearly useful to get a better information of the real clinical activity of each team and cancer center. To sum up, this tool can be useful to unifique and compare the different complexity in the clinical activity of Medical Oncology Teams, units and hospitals allocating resources based on this new system.
\end{abstract}

Keywords: Cancer Center, Clinical Activity, Inpatient, Outpatient, Working Load

\section{Introduction}

Increasingly, the field of oncology is focused on the implementation of strategies to improve care with an emphasis on evaluation. However, this process of implementation and evaluation is not easy so far. Clinical practice guidelines (CPGs) and quality metrics are the key tools to improve and assure quality cancer care [1],

Specifically, the daily activity of the Medical Oncology Department in a comprehensive oncological center is divided into three fundamental aspects: clinical assistance, teaching and research.

About teaching is clear how you can get an evaluation with academic results in degree and postdegree as doctoral thesis or master. Also the research projects are frankly reflected in the annual publications in scientific journals indexed with associated impact factor.

On the other hand, there is no generalized way [7], of evaluating clinical care activity in cancer centers in order to know the number of Medical Oncologist that are sufficient to reach the high quality you need on your department.

Minimal progress has been made in developing meaningful measures for cancer care, in part because of the complexity of the disease. Cancers vary greatly depending on location, type, stage, and molecular and genetic characteristics. Treatment may involve medical, surgical, and radiation oncologists. Similarly, most cancer treatment is delivered as outpatient care, which has been underrepresented in efforts to develop measures. These factors underlie the formidable challenge of representing a disparate set of diseases with a uniform set of 
quality measures [2].

There are scarce reported articles where it is described the characteristics of patients with cancer and their visits to outpatient, office-based physicians. One of them showed it is unclear whether shorter visit times impact the quality of medical care provided or whether physicians in these settings have become more proficient in caring for their patients [3].

In adittion, a pilot study from a single spanish oncology service [4]. was carry out in order to determine the real time each patient requires from a physician and thus establish a recommendation on the number of medical oncologists necessary the time required to care for a cancer patient: one oncologist for every 83 new patients annually. This number varies essentially during the evolution of the disease and differs according to the type of tumour. In general, without mentioning the complexity of the type of patient.

According to the previous in Spanish group it has been developed a new system based on the daily real activity of each medical oncologist of his team graduated according to the level of complexity of the activity [5]. The objective of the study is to describe health care in a comprehensive oncological center with the new method to identify new tools that help in a better management of available human resources, in order to better clinical care.

\section{Materials and Methods}

From January 2016 to December 2017 it was measured at a single Spanish cancer center, an individual assessment for each medical oncologist in their daily work in the following activities, according to the estimated average time in each of the activities in a clinical service of Medical Oncology.

First, the clinical activity of each unit has been calculated by adding the visits of patients attended. The outpatient clinical activity (ACA) is divided in turn according to the type of visit the patient performs: follow-up visit, treatment visit, clinical trial visit and first time visit.

Secondly, the overall clinical activity (OCA) is defined as the sum of the ambulatory clinical activity and the care of admitted patients

Moreover, in the First White Paper of the Medical Oncology in Spain (SEOM) of 2008, it have been updated the estimation on average duration of each type of activity assistance [6]. (see table 1)

Table 1. Visit type.

\begin{tabular}{ll}
\hline Visit type & Time recommended (min) \\
\hline Follow-up visit & 15 \\
Treatment visit & 30 \\
Clinical Trial & 45 \\
First visit/ Admitted & 60 \\
\hline
\end{tabular}

In addition, applying a weighting factor to them, it has been considered different values (from 1 to 4 ) depending on the complexity of the clinical activity: follow-up visit (1), treatment visit (2), Clinical trial visit or inpatient visit (3) and visit of new patient (4).

To these values it was assigned an equivalence with the times proposed by the SEOM to each type of clinical visit: 1 : $15 \mathrm{~min}$; 2:30 min; 3:45 min; 4: $60 \mathrm{~min}$, respectively.

In order to apply the activity system adjusted by previously described factor, it was added all the daily values of each medical oncologist.

The activity was not differentiated at the individual oncologist level, it was grouped by the functional oncological units of the center:

1. Gastrointestinal, Sarcoma, Head\&Neck tumor unit (GI unit)

2. Breast tumor unit (BREAST unit)

3. Genitourinary and melanoma tumor unit (GU unit)

4. Thoracic and Neuroncology unit (LUNG unit)

Subsequently, to calculate the overall adjusted activity (OAA) of each unit, it was divided by the number of medical oncologists in the unit (1-full time, 0.5-part time oncologist).

The assigned values are not arbitrary and obey expert consensus as previously described (10). For example, visiting the admitted patient, especially the newly admitted patient with medical complications associated with their oncological process, will require a comprehensive and multidisciplinary diagnostic/therapeutic approach in most cases that justifies investing more time than a patient who comes to consultation review on an outpatient basis.

Finally, in order to achieve internal validity, we obtain the data according to the traditional method and the new method in the same population

\section{Results}

In the cancer center from January 2016 to December 2017, there were 6418 new outpatients: 3156 in 2016 and 3262 in 2017. A total of 33197 and 34937 patients in 2016 and 2017 were attended in oncological center, respectively (figure 1).

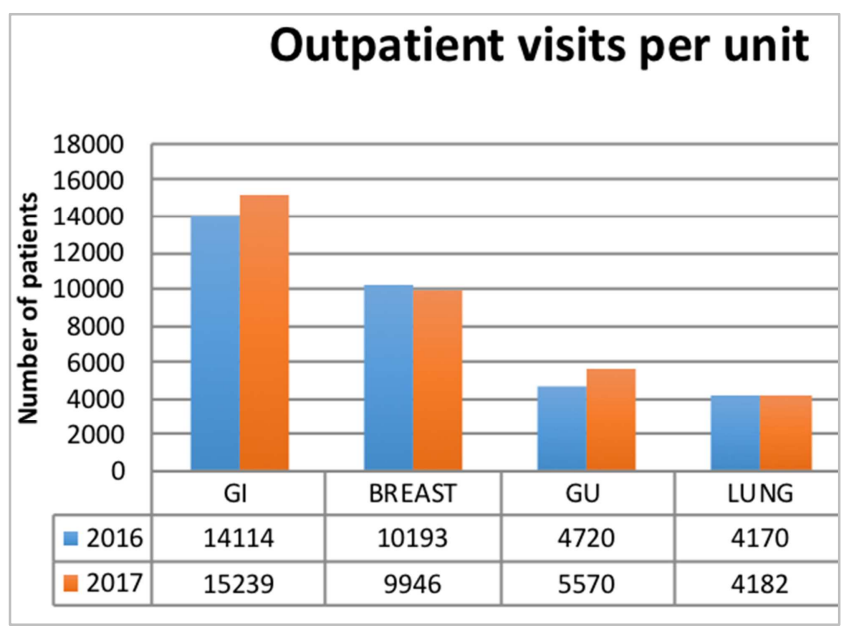

Figure 1. Number of outpatient visits per unit. GI: Gastrointestinal tumor Unit; BREAST: Breast tumor unit; GU: Genitourinary tumor unit; LUNG: Lung \& Neurooncology tumor unit.

The unit where more ACA were recorded was in GI unit (29353 patients, 44\%) followed by the breast unit (20139 patients, 28\%). GU unit attended 10290 patients (16\%) and 
LUNG unit 8352 patients (12\%) (figure 2)

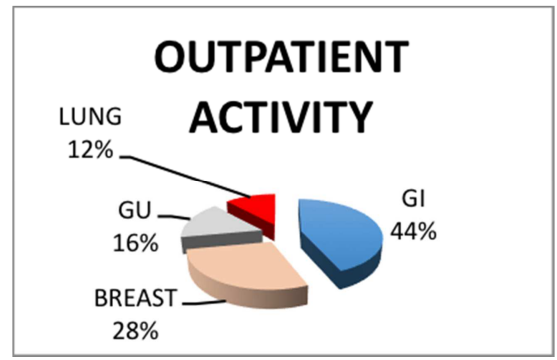

Figure 2. Outpatient activity by functional unit. GI: Gastrointestinal tumor Unit; BREAST: Breast tumor unit; GU: Genitourinary tumor unit; $L U N G$ : Lung \& Neurooncology tumor unit.

Regard to OCA the unit where more patients were treated was again in GI unit (47\%) followed by the breast unit (24\%). GU unit and LUNG unit obtained similar activity data (16\% and $13 \%$, respectively) (figure 3 )

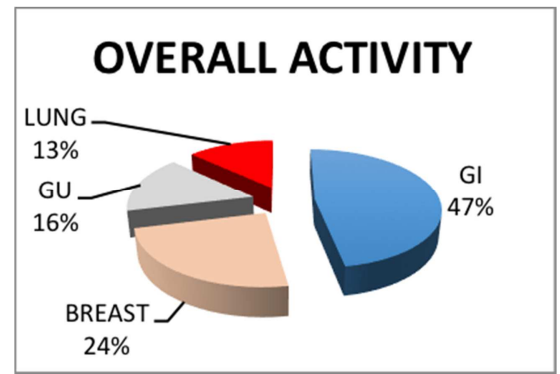

Figure 3. Overall activity (including the care activity of outpatients and inpatients) by functional unit: GI: Gastrointestinal tumor Unit; BREAST: Breast tumor unit; GU: Genitourinary tumor unit; LUNG: Lung \& Neurooncology tumor unit.
If we introduce a correction factor according to the complexity of care, the OAA, each oncology unit changed clearly when we applied the new method (Figure 4). For example, breast cancer unit change from $24 \%$ to $21 \%$ of the global activity, GI Unit from $47 \%$ to $49 \%$, Lung Unit from $13 \%$ to $14 \%$. In Genitourinary and Gynecological cancer and Prostate tumor units there are no change.

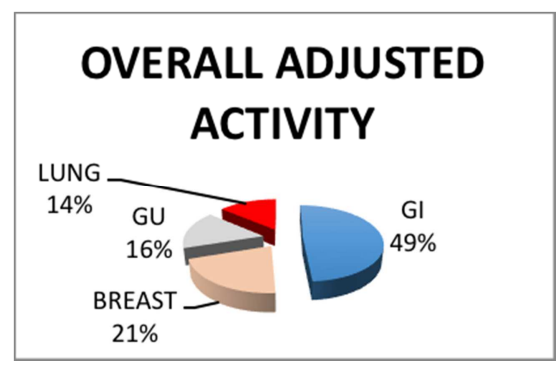

Figure 4. Adjusted general activity (overall care activity adjusted for complexity correction factor: follow-up visit of a patient (1), treatment visit (2), Clinical trial visit or inpatient visit (3) and visit of new patient (4)) functional unit: GI: Gastrointestinal tumor unit; MAMA: breast tumor unit; GU: genitourinary tumor unit; LUNG: tumor unit of lung and neurooncology.

Finally, if we calculate the working load adjusted by number of oncologists per unit and per month, again the Oncology GI Unit (742), is the unit with the highest workload during the analyzed period, followed closely by the unit of GU (617) and BREAST unit (549).

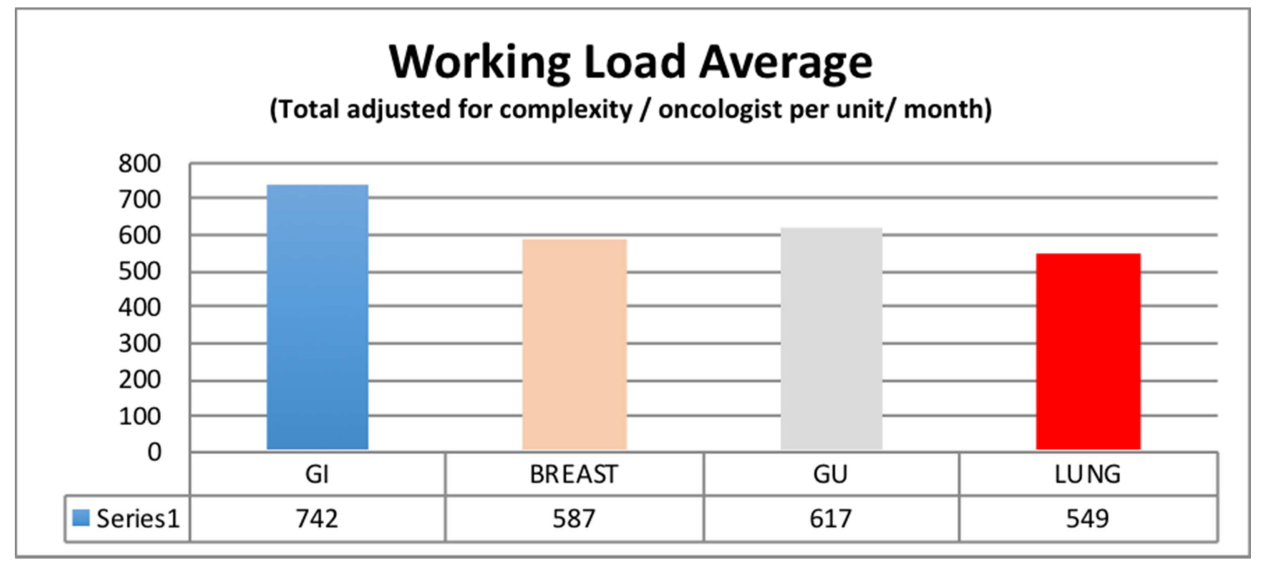

GI: Gastrointestinal tumor Unit; BREAST: Breast tumor unit; GU: Genitourinary tumor unit; LUNG: Lung \& Neurooncology tumor unit.

Figure 5. Working Load-Average: Total adjusted for complexity / oncologist per unit/ month.

\section{Discussion}

The new method for measuring clinical activity in a cancer center defines better oncological daily care than traditional methods [6]. since such methods are based on more general time criteria without considering type of patient visit (for example, the patient in a clinical trial), even though assuming different volumes of patients to estimate the value and clinical activity could be more accurate.

Although in a single care center, the different group methods by the functional oncological units might affect the finial value and clinical activity, it is important to remark a personalized clinical attention [8] to the oncological patient, particularly one of the cornerstone is to classify the patient according to the histological type and at the same time differentiate the type of visit to which the patient comes to 
the clinical center.

Obtaining the values by the method adjusted for the type of visit and the number of oncologists, the workload of each unit is more precisely defined so that the available resources can be distributed and managed more efficiently and that they can benefit more to the clinical attention of cancer patients. For example, in a cancer center, the BREAST unit has a higher volume of activity than the GU unit, but when adjusting the activity for the types of visit and number of oncologists, the workload is greater in GU unit than BREAST unit.

On the other hand, it is important to highlight the variability of the method within the same type of cancer, given the complexity of the clinical care that is linked to each patient.

These changes draw the different complexity of each Oncology Unit. Each patient with different tumor subtypes presents different healthcare needs inherent in the biological evolution of their cancer. The medical complications that patients present differs from the tumor type and this will be reflected in different number of outpatient visits or income. For example, patients with digestive or lung tumors present a higher rate of medical complications, requiring more time of admission and requiring a greater amount of resources.

Obviously this methodology has several shortcomings. First, well-defined prospective studies are needed in order to obtain firm conclusions about which methodology has the best results when evaluating the care activity in a cancer center.

There are also limitations at the time of reproducibility and external validation with this methodology in a generic way in other cancer centers due to the differences in the organization of patient care.

\section{Conclusion}

Furthermore, given the constant evolution in the field of cancer research, tools that can better quantify the type of assistance received by cancer patients are needed to better adjust the available resources and offer optimal clinical assistance [9]. In conclusion, it is clearly useful to get a better information of the real clinical activity of each team and cancer center to improve every day in daily clinical practice.

\section{Funding/Support}

None reported.

\section{Financial Disclosure}

None reported.

\section{Ethical Standards}

The study followed the Declaration of Helsinki guidelines.

\section{References}

[1] NCCN clinical guidelines. ESMO clinical guidelines.

[2] Spinks TE, Walters R, Felley TW et al. Improving Cancer Care Through Public Reporting of Meaningful Quality Measures. Health Affairs 2011; 30, 4:664-672.

[3] Guy PG, Richardson LC. Visit duration for outpatient physician office visit among patients with cancer. $J$ Oncol Pract 2012 May; 8(3 Suppl): 2s-8s.

[4] Salvador J, Grávalos C, Albanell J et al. Pilot study on workload estimate in breast cancer, lung cancer and colorectal cancer in a Medical Oncology Service at Valme hospital. Clin Transl Oncol 2012; 14:820-826.

[5] Alvarez R, Rodriguez J, Ugidos L et al. How to measure faculty clinical activity in a Comprenhensive Cancer Center? $J$ Clin Oncol 2017; 35: (suppl; abstr e18202).

[6] Colomer R, Alba E, Llombart A et al (2008) White Paper on Medical Oncology in Spain. Care Planning of Medical Oncology in Spain. Madrid: Dispublic, 2007.

[7] Donabedian A: Evaluating the quality of medical care. Milbank Mem Fund Q 44:166-206, 1966 (suppl 1).

[8] SJ, Calnan M: Convergence and divergence: Assessing criteria of consumer satisfaction across general practice, dental and hospital care settings. Soc Sci Med 33:707- 716, 1991.

[9] Goldzweig G, Meirowitz A, Hubert A, et al. Meeting expectations of patients With cancer: Relationship between patient satisfaction, depression, and coping. J Clin Oncol 2010. Published Ahead of Print on February 22, 2010 as 10.1200/JCO.2009.25.4987

[10] Strategy in Cancer of the National Health System 2006. Quality Plan for the National System of Health Ministry of Health and Consumption. Madrid, Spain. 